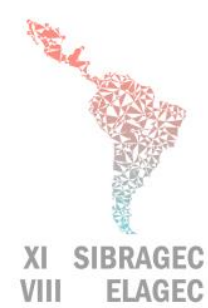

XI SIMPÓSIO BRASILEIRO DE GESTÃO E ECONOMIA DA CONSTRUÇÃO

VIII ENCUENTRO LATINOAMERICANO DE GESTIÓN Y ECONOMÍA DE LA CONSTRUCCIÓN

Do conhecimento à ação: práticas avançadas de gestão da produção

Londrina, Paraná, Brasil. 23 a 25 de Outubro de 2019

\title{
IDENTIFICAÇÃO DE PERDAS POR MAKING-DO E SEUS IMPACTOS EM CANTEIROS DE OBRAS GOIANOS
}

\author{
AMARAL, Tatiana G. (1); ELIAS, Karinny (2); BRANDÃO, Camila Mariana (3); BRAGA, \\ Pedro Boaratti (4)
}

(1) Universidade Federal de Goiás, (62) 981680902, e-mail: tatiana_amaral@ hotmail.com (2) Universidade Federal de Goiás, e-mail: karinnyelias22@gmail.com (3) Universidade Federal de Goiás, e-mail: c.mariana.b@gmail.com (4) Universidade Federal de Goiás, e-mail: pedrobraga.engcivil@gmail.com

\begin{abstract}
The making-do stands out for representing a potential generator of other wastes, such as safety reduction, quality problems, productivity and rework. The paper seeks to analyze the production-related wastes by making-do in order to identify, through the categories of improvisation wastes, the impacts generated by them, from the perspective of the Goiás scenario. At the end of the analysis a matrix was applied for risk analysis of making-do wastes. Data were collected from three construction companies in the city of Goiânia. For data collection, data triangulation was performed through the results of interview analysis, direct non-participant observations, photographic records and document analysis. After data analysis, it is concluded that most improvisations are related to short term planning, logistics and projects. However, the most important impacts of these types of wastes are the need for rework and material waste. As a result, we highlight the importance of studying the wastes related to making-do, as it goes beyond the waste of materials, highlighting issues related to health and safety of the workforce and impacts on the production process as a whole.
\end{abstract}

Keywords: Waste; Making-do; Improvisation.

\section{INTRODUÇÃ̃O}

Na construção civil, a medição das perdas se referia apenas ao consumo excessivo de materiais (BOSSINK; BROUWERS, 1996; ENSHASSI, 1996). Ampliando essa visão, Formoso et al. (2002) propuseram uma nova classificação de perdas, as de outras naturezas. Dessa forma, incluindo as perdas de materiais e as sete propostas por Ohno (1988), tem-se: superprodução, espera, transporte, processamento, estoque, movimento, retrabalho, substituição e perdas de outras naturezas.

Viana, Formoso e Kalsaas (2012) por sua vez, sintetizam estudos sobre diferentes tipos de perdas (Quadro 1).

Além das perdas classificadas por Formoso (2002), foi identificada a perda por making-do, que se destaca por representar uma potencialidade de geração de outras perdas como redução da segurança, problemas de qualidade, work in progress e retrabalho (SOMMER, 2010; FORMOSO et al., 2011; FIREMAN et al., 2013).

Santos, Santos (2017) destacaram uma relação entre as perdas associadas ao making-do e o sistema de gestão. As perdas analisadas, em cinco canteiros de obras, apresentaram relação causal com falhas da gestão de qualidade e deficiência do planejamento e controle de produção (PCP), principalmente no sequenciamento de atividades. 
Em Goiânia, o levantamento de perda por making-do se integra a um grupo de pesquisa, no qual foram levantados dados em oito empresas construtoras goianas para identificar a presença de perdas por making-do e seus impactos (BRANDÃO e ELIAS, 2018; AMARAL, MARTINS, DINOAH e RIBEIRO, 2018 e BRAGA, 2018).

\section{Quadro 1 - Estudos sobre diferentes tipos de perdas}

\begin{tabular}{|l|l|}
\hline \multicolumn{1}{|c|}{ AUTORES } & ESTUDOS \\
\hline SKOYLES (1976) & \\
BOSSINK e BROUWERS (1996) & Excesso no consumo de materiais \\
ENSHASSI (1996) & \\
FORMOSO et al. $(2002)$ & \\
NAHMENS e IKUMA (2011) & \\
CHOI et al. (2002) & \\
LOVE e EDWARDS (2004) & \\
KOSKENVESA et al. (2008) & \\
SENARATNE e SEXTON (2009) & \\
YU et al. (2009) & Atividades que não agregam valor e \\
BULHÕES (2009) & retrabalhos \\
HWANG et al. (2009) & \\
ZHAO et al. (2010) & \\
HAN et al. (2011) & \\
NAHMENS e MULLENS (2011) & \\
\hline BURATI, FARRINGTON e LEDBETTER & Desvio da qualidade \\
(1992) & Não conhecimento das necessidades \\
\hline WOMACK e JONES (2004) & do cliente \\
\hline MONDEN (1983) & Investimento de capital desnecessário \\
\hline BOSSINK e BROUWERS (1996) & Roubo ou vandalismo \\
\hline $\begin{array}{l}\text { KOSKELA (2004) } \\
\text { SOMMER (2010) }\end{array}$ & Making- do \\
\hline
\end{tabular}

Fonte: Adaptado de Viana, Formoso e Kalsaas (2012).

O trabalho apresenta os resultados parciais deste grupo, e tem como objetivo analisar as perdas por making-do relacionadas à produção na perspectiva do cenário goiano. Como objetivo secundário foi analisado os riscos relacionados a estas perdas.

\section{MAKING DO: CONCEITOS E DEFINIÇÕES}

Koskela (2004) define making-do como a perda que ocorre quando uma tarefa inicia sem todas as suas entradas, ou quando ela tem sua execução continuada, mesmo com a cessão de uma ou mais entradas. Observa que o modelo de gestão está todo voltado para a tarefa iniciada, ao passo que as discrepâncias entre o que foi planejado e a realidade de canteiro não são levados em conta, resultando em situações nas quais o making-do surge como única solução para que o trabalho não seja interrompido.

A partir dos estudos de Koskela (2000), Sommer (2010) e Fireman (2012), foram identificadas algumas categorias de perdas por making-do, pré-requisitos necessários e possíveis impactos gerados 
por elas. Sommer (2010) apresentou sete categorias de perdas e Fireman (2012) acrescentou o sequenciamento, totalizando assim, oito categorias de perdas por making-do (Quadro 2).

Autores como Koskela (2004) e Sommer (2010) estudaram alguns impactos causados pelas perdas já apresentadas, em conjunto com estes, Fireman (2012) acrescentou um impacto denominado falta de terminalidade.

Quadro 2 - Classificação de perdas por making-do

\begin{tabular}{|c|c|c|c|c|c|}
\hline $\begin{array}{c}\text { IDENTIFICAÇÃO } \\
\text { / CATEGORIA }\end{array}$ & AUTOR & $\begin{array}{c}\text { PRÉ CONDIÇÃO / } \\
\text { PRÉ REQUISITO } \\
\text { AUSENTE } \\
\end{array}$ & AUTOR & $\begin{array}{c}\text { IMPACTO / } \\
\text { AVALIAÇÃO }\end{array}$ & AUTOR \\
\hline Acesso/ mobilidade & $\begin{array}{l}\text { Sommer } \\
(2010)\end{array}$ & Informação & $\begin{array}{c}\text { Sommer } \\
(2010) \\
\text { Koskela } \\
(2000)\end{array}$ & Baixa produtividade & $\begin{array}{l}\text { Sommer } \\
(2010)\end{array}$ \\
\hline $\begin{array}{c}\text { Ajuste de } \\
\text { componentes }\end{array}$ & $\begin{array}{l}\text { Sommer } \\
(2010)\end{array}$ & $\begin{array}{c}\text { Materiais e } \\
\text { componentes }\end{array}$ & $\begin{array}{c}\text { Sommer } \\
(2010) \\
\text { Koskela } \\
(2000) \\
\end{array}$ & $\begin{array}{l}\text { Diminuição da } \\
\text { qualidade }\end{array}$ & $\begin{array}{c}\text { Sommer } \\
(2010)\end{array}$ \\
\hline Área de trabalho & $\begin{array}{l}\text { Sommer } \\
(2010)\end{array}$ & Mão de obra & $\begin{array}{c}\text { Sommer } \\
(2010) \\
\text { Koskela } \\
(2000) \\
\end{array}$ & Retrabalho & $\begin{array}{c}\text { Sommer } \\
(2010)\end{array}$ \\
\hline $\begin{array}{l}\text { Armazenamento: } \\
\text { estoque de materiais } \\
\text { ou componentes }\end{array}$ & $\begin{array}{l}\text { Sommer } \\
(2010)\end{array}$ & $\begin{array}{l}\text { Equipamentos/ } \\
\text { Ferramentas }\end{array}$ & $\begin{array}{c}\text { Sommer } \\
(2010) \\
\text { Koskela } \\
(2000) \\
\end{array}$ & Perdas de materiais & $\begin{array}{c}\text { Sommer } \\
(2010)\end{array}$ \\
\hline $\begin{array}{l}\text { Equipamentos / } \\
\text { ferramentas }\end{array}$ & $\begin{array}{l}\text { Sommer } \\
(2010)\end{array}$ & Espaço & $\begin{array}{c}\text { Sommer } \\
(2010) \\
\text { Koskela } \\
(2000)\end{array}$ & $\begin{array}{l}\text { Redução da } \\
\text { segurança }\end{array}$ & $\begin{array}{l}\text { Sommer } \\
(2010)\end{array}$ \\
\hline $\begin{array}{c}\text { Instalação provisória: } \\
\text { suprimento de água e } \\
\text { eletricidade }\end{array}$ & $\begin{array}{l}\text { Sommer } \\
(2010)\end{array}$ & Serviços Interligados & $\begin{array}{c}\text { Sommer } \\
(2010) \\
\text { Koskela } \\
(2000) \\
\end{array}$ & Desmotivação & $\begin{array}{l}\text { Sommer } \\
(2010)\end{array}$ \\
\hline Proteção & $\begin{array}{l}\text { Sommer } \\
(2010)\end{array}$ & Condições Externas & $\begin{array}{c}\text { Sommer } \\
(2010) \\
\text { Koskela } \\
(2000) \\
\end{array}$ & $\begin{array}{c}\text { Falta de } \\
\text { terminalidade }\end{array}$ & $\begin{array}{l}\text { Fireman } \\
(2012)\end{array}$ \\
\hline Sequenciamento & $\begin{array}{l}\text { Fireman } \\
(2012)\end{array}$ & $\begin{array}{c}\text { Instalações: } \\
\text { infraestrutura do } \\
\text { espaço de trabalho }\end{array}$ & $\begin{array}{l}\text { Sommer } \\
(2010)\end{array}$ & & \\
\hline
\end{tabular}

Fonte: Adaptado de Koskela (2000), Sommer (2010) e Fireman (2012). 


\section{MÉTODO}

\subsection{Classificação da pesquisa}

Com relação à abordagem, a pesquisa se classifica como quantitativa e qualitativa, por analisar os resultados numéricos e apresentar significados, sentidos e interpretações teóricas sobre as perdas identificadas.

Quanto à natureza, a pesquisa é aplicada, pois tem como um dos objetivos principais a produção de conhecimento a ser utilizado na construção civil.

Com relação aos objetivos e procedimentos, a pesquisa é classificada como exploratória.

\subsection{Caracterização das empresas e seus empreendimentos}

Os dados foram levantados em um empreendimento de três empresas construtoras durante cinco meses em 2018. A caracterização dessas empresas está apresentada no Quadro 3 e dos empreendimentos no Quadro 4.

\section{Quadro 3 - Caracterização das empresas participantes}

\begin{tabular}{|c|c|c|c|c|c|}
\hline Empresa & Certificações & $\begin{array}{c}\text { Tempo de } \\
\text { atuação no } \\
\text { mercado }\end{array}$ & $\begin{array}{c}\text { Obras em andamento } \\
\text { no primeiro semestre } \\
\text { de } 2018\end{array}$ & $\begin{array}{c}m^{2} \text { em execução } \\
\text { em } 2018\end{array}$ & $\begin{array}{l}\text { Porte da } \\
\text { empresa }\end{array}$ \\
\hline \multirow[b]{3}{*}{ A } & $\begin{array}{l}\text { ABNT NBR ISO 14001:2004- } \\
\text { Sistema de Gestão Ambiental }\end{array}$ & \multirow[b]{3}{*}{21 anos } & $\begin{array}{l}01 \text { empreendimento } \\
\text { residencial de médio } \\
\text { padrão }\end{array}$ & $47.789,71$ & \multirow[b]{3}{*}{ Grande porte } \\
\hline & ABNT NBR ISO 9001:2008 & & 01 faculdade & $11.063,00$ & \\
\hline & $\begin{array}{l}\text { OHSAS 18001:2007 - Sistema de } \\
\text { Gestão da Saúde e Segurança do } \\
\text { Trabalho } \\
\text { PBQP-H NÍVEL A - Sistema de } \\
\text { Gestão da Qualidade } \\
\end{array}$ & & $\begin{array}{c}03 \text { EHIS (um em } \\
\text { Goiânia e dois no Rio de } \\
\text { Janeiro) }\end{array}$ & $\begin{array}{r}12.493,00 \mathrm{GO} \\
12.595,01 \mathrm{RJ} \\
26.001,22 \mathrm{RJ}\end{array}$ & \\
\hline \multirow{4}{*}{ B } & ABNT NBR ISO 9001:2008 & \multirow{4}{*}{19 anos } & $\begin{array}{l}01 \text { empreendimento } \\
\text { residencial de médio }\end{array}$ & $20.853,13$ & \multirow{4}{*}{ Grande porte } \\
\hline & & & 02 empreendimentos & $23.219,83$ & \\
\hline & \multirow{2}{*}{$\begin{array}{l}\text { PBQP-H NÍVEL A - Sistema de } \\
\text { Gestão da Qualidade }\end{array}$} & & residenciais de alto & $24.796,84$ & \\
\hline & & & 01 hotel + residencial & $19.572,45$ & \\
\hline & ABNT NBR ISO 9001:2015 & \multirow[b]{2}{*}{32 anos } & \multirow{2}{*}{$\begin{array}{l}02 \text { empreendimentos } \\
\text { residenciais de médio } \\
\text { padrão }\end{array}$} & \multirow[b]{2}{*}{$\begin{array}{l}27.169,88 \\
29.279,84\end{array}$} & \multirow[b]{2}{*}{ Grande porte } \\
\hline $\mathrm{C}$ & $\begin{array}{l}\text { PBQP-H NÍVEL A - Sistema de } \\
\text { Gestão da Qualidade }\end{array}$ & & & & \\
\hline
\end{tabular}

Fonte: Autoria própria. 
SIBRAGEC - ELAGEC 2019 - de 23 a 25 de Outubro - LONDRINA - PR

Quadro 4 - Caracterização das obras

\begin{tabular}{|c|c|c|c|c|c|c|}
\hline Obra & Pavimentos & $\begin{array}{c}\text { Número de } \\
\text { torres }\end{array}$ & $\begin{array}{c}\text { Fases em execução no } \\
\text { período em estudo }\end{array}$ & $\begin{array}{c}\mathbf{m}^{2} \text { de } \\
\text { construção }\end{array}$ & Mão de obra & $\begin{array}{c}\text { Data de entrega } \\
\text { prevista }\end{array}$ \\
\hline \multirow{4}{*}{ A } & 03 subsolos & \multirow{4}{*}{2} & Alvenaria & \multirow{4}{*}{$47.789,71$} & \multirow{4}{*}{$\begin{array}{c}\text { Maioria } \\
\text { Tercerizada }\end{array}$} & \multirow{2}{*}{ Torre A - Outubro 2018} \\
\hline & 01 térreo & & Instalações & & & \\
\hline & 27 pavimentos tipo & & Esquadrias & & & \multirow{2}{*}{ Torre B - Abril/2018 } \\
\hline & 03 Penthouses / 01 cobertura & & Acabamentos & & & \\
\hline \multirow{5}{*}{ B } & 03 subsolos & \multirow{5}{*}{1} & Estrutura & \multirow{5}{*}{$20.853,13$} & \multirow{5}{*}{$\begin{array}{c}\text { Maioria } \\
\text { Tercerizada }\end{array}$} & \multirow{5}{*}{ Outubro/2018 } \\
\hline & 01 térreo & & Alvenaria & & & \\
\hline & 01 pavimento de garagem/lazer & & Instalações & & & \\
\hline & 31 pavimentos tipo & & Esquadrias & & & \\
\hline & 01 ático & & Acabamentos & & & \\
\hline \multirow{5}{*}{$\mathrm{C}$} & 01 subsolo & \multirow{5}{*}{1} & Estrutura & \multirow{5}{*}{$27.169,88$} & \multirow{5}{*}{$\begin{array}{c}\text { Tercerizada e } \\
\text { Própria }\end{array}$} & \multirow{5}{*}{ Outubro/2019 } \\
\hline & 01 térreo & & \begin{tabular}{|l|} 
Alvenaria \\
\end{tabular} & & & \\
\hline & 03 pavimentos de garagem & & Instalações & & & \\
\hline & 01 pavimento de lazer & & \begin{tabular}{|l|} 
Esquadrias \\
\end{tabular} & & & \\
\hline & 28 pavimentos tipo & & Acabamentos & & & \\
\hline
\end{tabular}

Fonte: Autoria própria.

\subsection{Levantamento e análise de dados}

A metodologia se baseia no método proposto por Sommer (2010) e Fireman (2012), considerando as definições de categorias, impactos e pré-requisitos propostos por estes autores.

Foi realizada uma triangulação dos dados, por meio dos resultados levantados nas entrevistas, observações diretas não participantes, registros fotográficos e análise documental.

As entrevistas foram norteadas pelo roteiro de perguntas (Apêndice A) adaptado do estudo de Santos e Santos (2017), as quais foram realizadas com engenheiros, estagiários, mestre de obras e encarregados, com a finalidade de identificar as origens e consequências das perdas por making-do.

As observações não participantes, foram consideradas como as principais fontes de evidências coletadas e foram realizadas por meio de visitas em campo.

Os registros fotográficos aumentaram a possibilidade de comprovação das informações levantadas.

Foram analisados documentos relacionados à gestão de produção, tais como, projetos executivos e de canteiro de obras, cronogramas dos empreendimentos, fichas de verificação de serviços, além de documentos relacionados ao planejamento e seus controles.

A classificação dos dados levantados se baseou na proposição de Sommer (2010) e Fireman (2012) (Quadro 5).

Os dados coletados foram padronizados em planilha eletrônica (Quadro 6). O item pré-requisitos ausentes da planilha de tabulação dos dados foi preenchido de acordo com as observações dos pesquisadores e nos relatos dos entrevistados. Nesse item, foi identificada a causa da perda por making-do de cada caso coletado, podendo ela ser única ou múltipla.

Além dos impactos já apresentados por Koskela (2004), Sommer (2010) e Fireman (2012), foram sugeridos pelos autores, custo e cronograma.

Foi ainda realizada uma análise de riscos das perdas por making-do, utilizando a metodologia proposta por Fireman (2012). O método proposto se baseia em uma avaliação subjetiva e qualitativa dos casos e permite o seu agrupamento em maior prioridade (vermelho), prioridade intermediária (amarelo) e menor prioridade (verde) (Quadro 7). 
SIBRAGEC - ELAGEC 2019 - de 23 a 25 de Outubro - LONDRINA - PR

Quadro 5 - Classificação dos dados

\begin{tabular}{|l|l|l|l|}
\hline \multicolumn{1}{|c|}{ PRÉ CONDIÇÕES } & \multicolumn{1}{|c|}{ CATEGORIAS } & \multicolumn{1}{c|}{ IMPACTOS } & OUTRAS PERDAS \\
\hline Condições Externas & Acesso/Mobilidade & Cronograma & $\begin{array}{l}\text { Elaboração de } \\
\text { produtos defeituosos }\end{array}$ \\
\hline Equipamentos/Ferramentas & Ajuste de Componentes & Custo & Espera \\
\hline Espaço & Área de Trabalho & Desmotivação & Estoque \\
\hline Informação & Armazenamento & Diminuição da Produtividade & Movimentação \\
\hline Instalações & Equipamentos/Ferramentas & Falta de Terminalidade & Processamento \\
\hline Mão de Obra & Instalações Provisórias & Perda de Material & Produto Defeituoso \\
\hline Materiais e Componentes & Proteção & Redução da Qualidade & Substituição \\
\hline Serviços Interdependentes & Sequenciamento & Redução da Segurança & Superprodução \\
\hline & \multicolumn{2}{|l|}{ Retrabalho } & Transporte \\
\hline
\end{tabular}

Fonte: Adaptado de Sommer (2010) e Fireman (2012).

\section{Quadro 6 - Banco de dados}

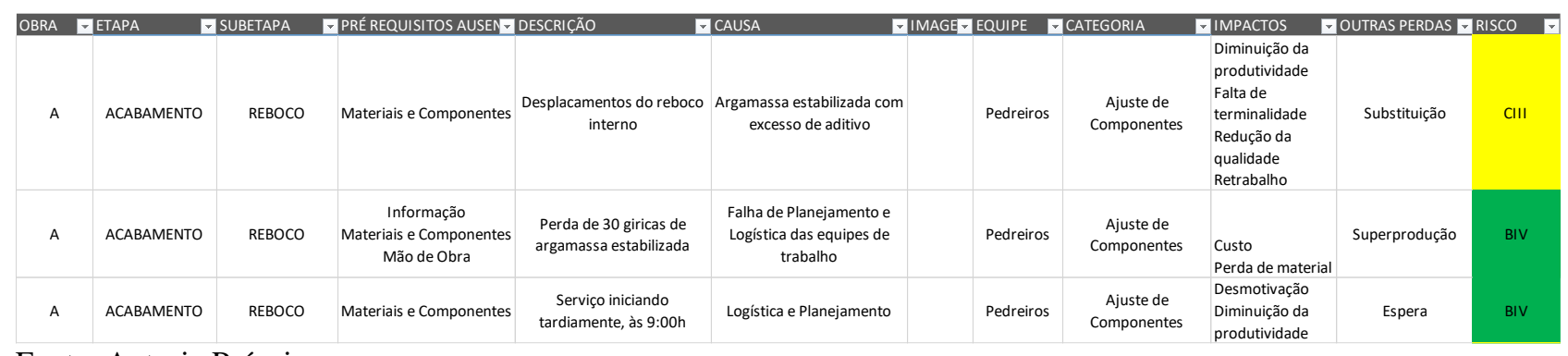

Fonte: Autoria Própria.

\section{Quadro 7 - Matriz para avaliação de riscos}

\begin{tabular}{|l|l|l|l|l|l|}
\hline \multirow{2}{*}{\multicolumn{1}{|c|}{ PROBABILIDADE }} & \multicolumn{5}{c|}{ SEVERIDADE } \\
\cline { 2 - 6 } & Muito Alta - I & Alta - II & Moderada - III & Baixa - IV & Muito Baixa - V \\
\hline A - improvável & & & & & \\
\hline B - extremamente remota & & & & & \\
\hline C - remota & & & & & \\
\hline D - provável & & & & & \\
\hline E - frequente & & & & & \\
\hline
\end{tabular}

Fonte: Fireman (2012).

Na análise é considerada a severidade (Quadro 8) e a probabilidade de repetição dos casos observados. A definição desses dois parâmetros foi fundamentada em momentos de brainstorming do grupo de pesquisa e equipe técnica das empresas. 
SIBRAGEC - ELAGEC 2019 - de 23 a 25 de Outubro - LONDRINA - PR

Quadro 8 - Critérios de severidade

\begin{tabular}{|c|c|}
\hline SEVERIDADE & IMPACTO \\
\hline \multirow{3}{*}{$\begin{array}{l}\text { Severidade muito } \\
\text { alta e alta }\end{array}$} & - Afetam significativamente a segurança dos colaboradores \\
\hline & $\begin{array}{l}\text { - Impactam financeiramente no orçamento e no cronograma } \\
\text { do empreendimento }\end{array}$ \\
\hline & $\begin{array}{l}\text { - Exigem decisões rápidas da equipe de gestão devido aos seus } \\
\text { impactos }\end{array}$ \\
\hline \multirow[t]{3}{*}{$\begin{array}{l}\text { Severidade } \\
\text { moderada }\end{array}$} & $\begin{array}{l}\text { - Não exigem decisões imediatas da equipe de gestão devido } \\
\text { aos seus impactos }\end{array}$ \\
\hline & $\begin{array}{l}\text { - Afetam em menores proporções o cronograma do } \\
\text { empreendimento }\end{array}$ \\
\hline & $\begin{array}{l}\text { - Afetam em menores proporções } \\
\text { empreendimento }\end{array}$ \\
\hline \multirow{3}{*}{$\begin{array}{l}\text { Severidade baixa e } \\
\text { muito baixa }\end{array}$} & - Casos de rápida e fácil resolução pela equipe de gestão \\
\hline & - Baixos impactos no cronograma do empreendimento \\
\hline & - Baixos impactos no orçamento do empreendimento \\
\hline
\end{tabular}

Fonte: Adaptado de Fireman (2012).

O critério de probabilidade foi definido de acordo com a quantidade de ocorrência das falhas registradas. Os casos com alta incidência foram definidos como frequente, os de frequência menor como provável, e os casos com foram distribuídos em improvável; extremamente remota e remota.

Como resultado do cruzamento dos critérios de severidade e probabilidade obteve-se o grau de prioridade das falhas coletadas (Quadro 7).

\section{RESULTADOS}

As categorias de maior ocorrência foram sequenciamento, ajuste de componentes e área de trabalho (Figura 1).

A partir da análise de risco, foi calculada a porcentagem de casos ocorridos para cada categoria identificados com maior prioridade, de acordo com classificação da matriz para avaliação de riscos (Figura 2).

A categoria equipamentos/ferramentas apareceu somente na obra $\mathrm{C}$, e, em apenas um caso de rompimento da tubulação de concreto.

No estudo realizado por Sommer (2010), a categoria Proteção aparece com maior índice de ocorrências, e com base nos resultados levantados e ilustrados na Figura 1, observa-se que esta categoria apareceu em quarto lugar. Por outro lado, as empresas devem intervir nos problemas relacionados a essa categoria, uma vez que pois $67 \%$ dos casos relacionados à proteção aparecem como maior prioridade, indicando um risco elevado à empresa (Figura 6). 
SIBRAGEC - ELAGEC 2019 - de 23 a 25 de Outubro - LONDRINA - PR

Figura 1 - Comparativo de categorias de perdas

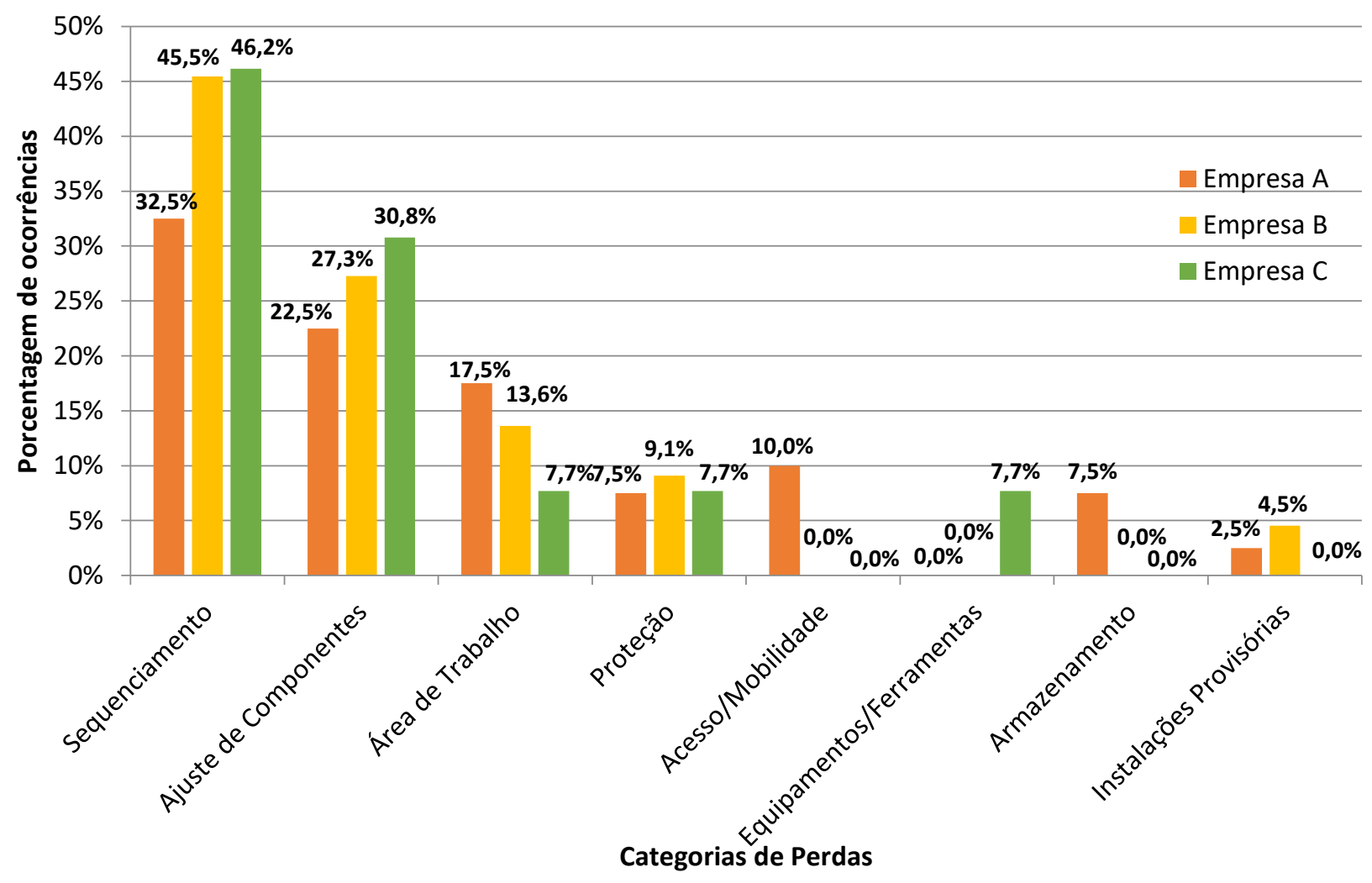

Fonte: Autoria própria.

Figura 2 - Porcentagem de casos por categoria com maior prioridade

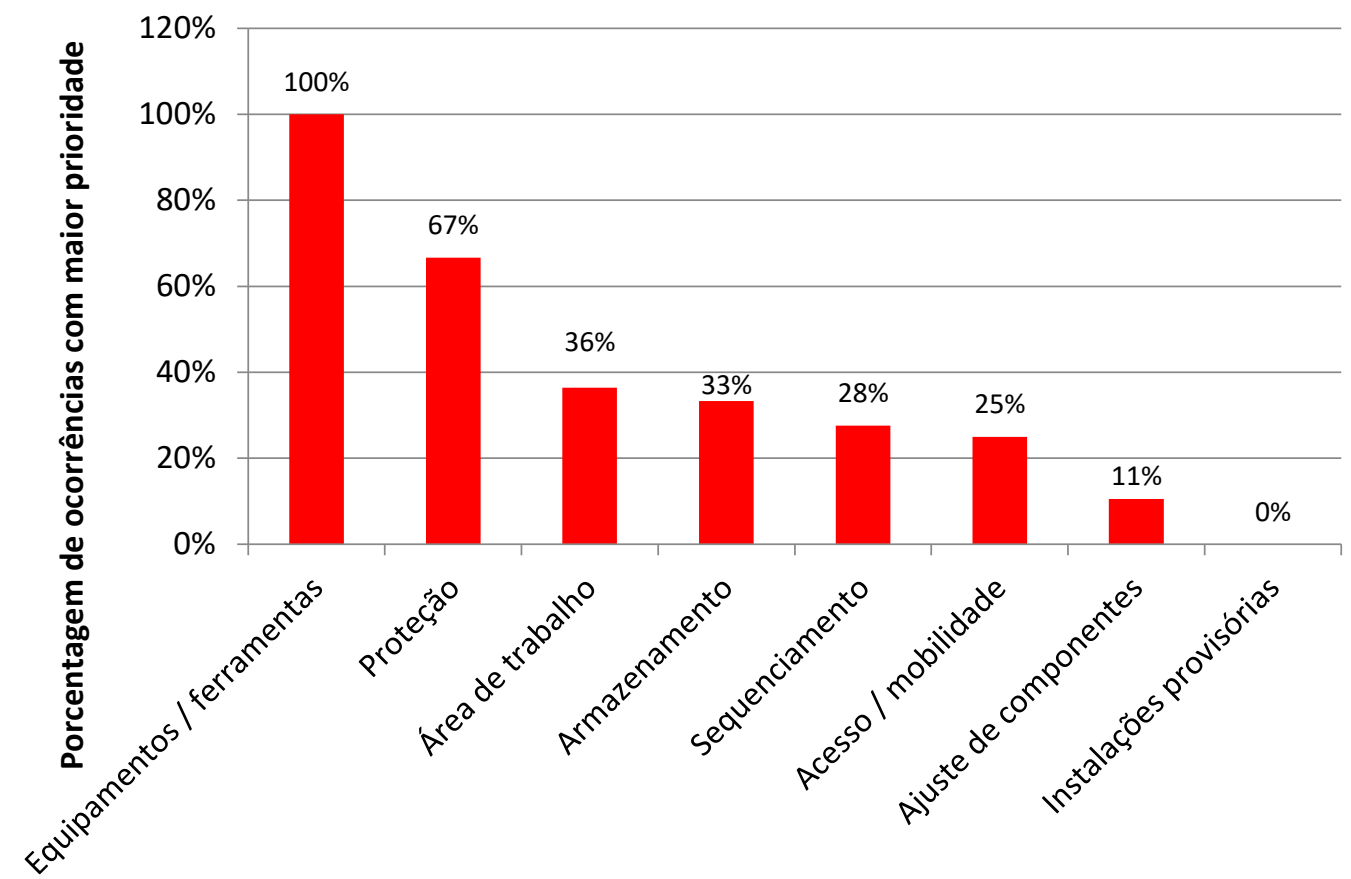

Categorias de perda

Fonte: Autoria própria. 
As perdas relacionadas à categoria de sequenciamento ocorreram devido ao atraso na definição dos apartamentos personalizados, falha na verificação de serviços finalizados e falta de acompanhamento durante a execução dos serviços, o que gerava impactos no cronograma, no custo, perda de material e retrabalho.

A Figura 3 apresenta a porcentagem de ocorrência dos pré-requisitos que apresentaram falhas. Destaca-se o item instalações por apresentar a menor ocorrência $(0,9 \%)$ nas obras estudadas.

O pré-requisito informação foi apontado como uma das principais origens das improvisações identificadas como ajuste de componentes, acesso/mobilidade, proteção e sequenciamento nos dados coletados. Em 27,3\% dos casos, relacionavam-se à falta de projetos específicos, incompatibilidade entre projetos e detalhamentos e/ou inexistência de treinamentos específicos dos funcionários.

O item materiais e componentes resultou em $21,8 \%$ das improvisações observadas, as quais ocorreram devido a falhas de logística no recebimento de materiais para realização do serviço ou inadequação quanto ao uso.

Em relação aos impactos das perdas por improvisação, foi observado que $24 \%$ dos casos resultam em retrabalho e, $15 \%$, geram perdas de material, evidenciando assim a relação entre estes dois impactos (Figura 4).

A partir do percentual de perdas, das falhas de pré-requisitos e dos possíveis impactos gerados, analisou-se a relação entre os indicadores. Destaca-se que cada caso pode apresentar mais de um prérequisito ou impacto, resultando em uma soma superior a 100\%. A matriz apresentada na Figura 5 relaciona o percentual de ocorrência de cada impacto por categoria de perdas.

Figura 3 - Porcentagem de ocorrências devido à falha nos pré-requisitos

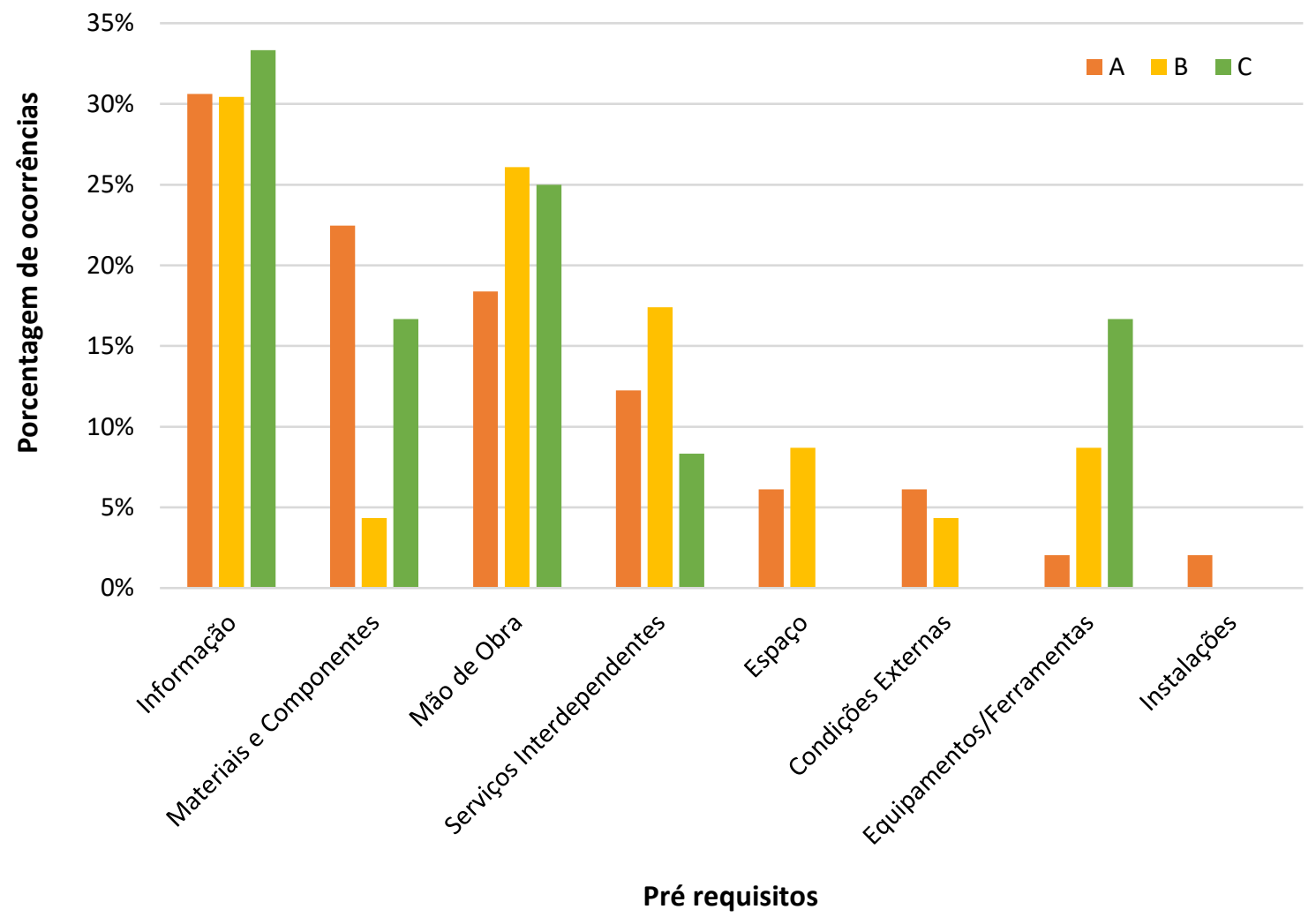

Fonte: Autoria própria. 
SIBRAGEC - ELAGEC 2019 - de 23 a 25 de Outubro - LONDRINA - PR

Figura 4 - Porcentagem de impactos gerados por improvisação

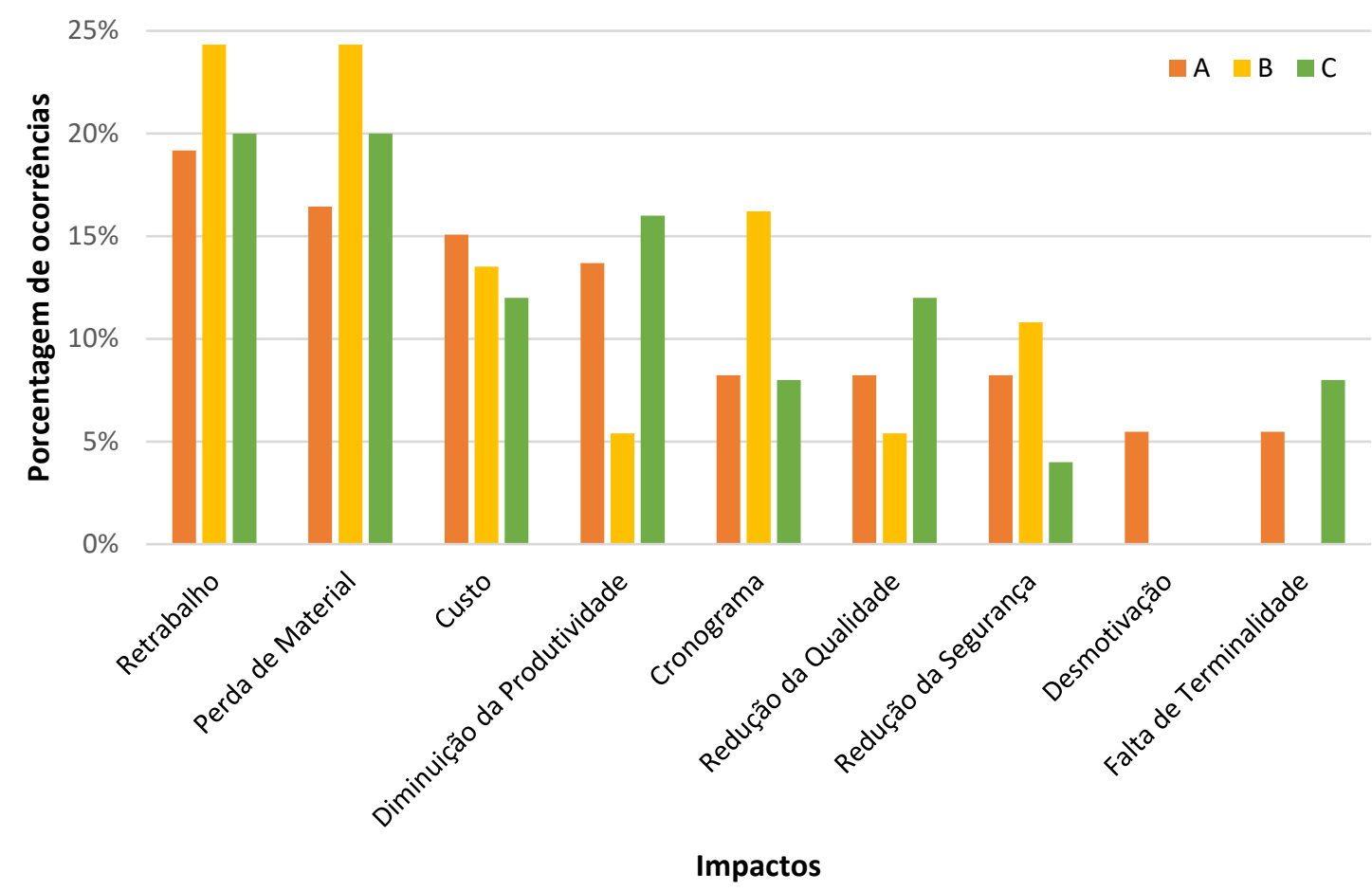

Fonte: Autoria própria.

Figura 5 - Matriz relação categoria $x$ impacto

\begin{tabular}{|c|c|c|c|c|c|c|c|c|c|}
\hline CATEGORIA X IMPACTO & $\begin{array}{l}\frac{\pi}{E} \\
\frac{1}{0} \\
\frac{0}{00} \\
\frac{0}{0} \\
\text { ப் }\end{array}$ & 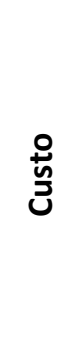 & 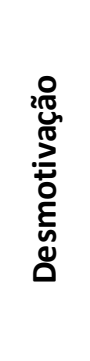 & 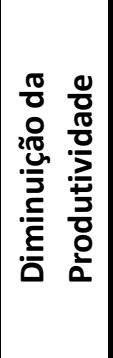 & 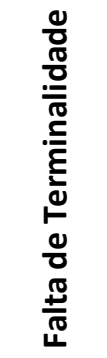 & $\begin{array}{l}\bar{\pi} \\
\frac{\pi}{2} \\
\mathbb{0} \\
\frac{ \pm}{0} \\
\sum \\
0 \\
\frac{0}{0} \\
\frac{\pi}{0} \\
\frac{0}{0} \\
0\end{array}$ & 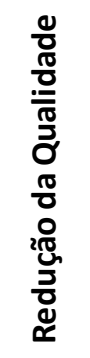 & 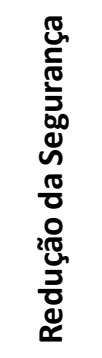 & 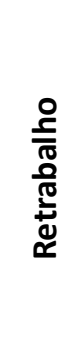 \\
\hline Acesso / mobilidade & $0 \%$ & $0 \%$ & $25 \%$ & $25 \%$ & $0 \%$ & $0 \%$ & $0 \%$ & $75 \%$ & $0 \%$ \\
\hline Ajuste de componentes & $21 \%$ & $37 \%$ & $11 \%$ & $32 \%$ & $11 \%$ & $26 \%$ & $5 \%$ & $0 \%$ & $68 \%$ \\
\hline Área de trabalho & $0 \%$ & $9 \%$ & $9 \%$ & $9 \%$ & $9 \%$ & $9 \%$ & $45 \%$ & $64 \%$ & $36 \%$ \\
\hline Armazenamento & $0 \%$ & $33 \%$ & $33 \%$ & $33 \%$ & $0 \%$ & $67 \%$ & $33 \%$ & $67 \%$ & $0 \%$ \\
\hline Equipamentos / ferramentas & $0 \%$ & $0 \%$ & $0 \%$ & $0 \%$ & $0 \%$ & $100 \%$ & $0 \%$ & $0 \%$ & $0 \%$ \\
\hline Instalações provisórias & $0 \%$ & $50 \%$ & $50 \%$ & $50 \%$ & $0 \%$ & $0 \%$ & $50 \%$ & $50 \%$ & $0 \%$ \\
\hline Proteção & $0 \%$ & $0 \%$ & $0 \%$ & $0 \%$ & $0 \%$ & $0 \%$ & $33 \%$ & $100 \%$ & $0 \%$ \\
\hline Sequenciamento & $31 \%$ & $38 \%$ & $3 \%$ & $34 \%$ & $17 \%$ & $55 \%$ & $14 \%$ & $0 \%$ & $79 \%$ \\
\hline
\end{tabular}

Fonte: Autoria própria.

Apesar da segurança não ser o impacto numericamente mais significativo das obras em estudo $(11,4 \%)$, tornando-se um ponto de alerta para as empresas, uma vez que o comprometimento da segurança apresenta um risco elevado. 
Ainda é possível analisar a relação entre as perdas e sua origem, evidenciando que muitas podem ser as causas para ocorrência de uma determinada perda, levando à improvisações (Figura 6).

Figura 6 - Matriz relação categoria x pré-requisitos

\begin{tabular}{|c|c|c|c|c|c|c|c|c|}
\hline $\begin{array}{l}\text { CATEGORIA X PRÉ- } \\
\text { REQUISITOS }\end{array}$ & 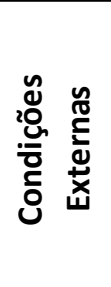 & 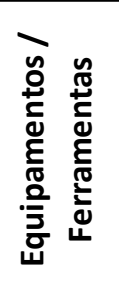 & $\begin{array}{l}\text { 울 } \\
\text { оू } \\
\text { 山ै }\end{array}$ & 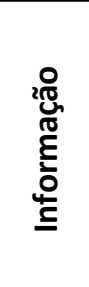 & 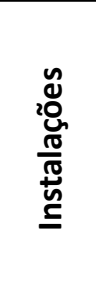 & $\begin{array}{l}\frac{5}{0} \\
0 \\
0 \\
\frac{0}{0} \\
0 \\
\Sigma\end{array}$ & 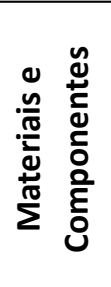 & 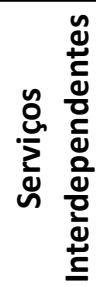 \\
\hline Acesso / mobilidade & $0 \%$ & $0 \%$ & $25 \%$ & $75 \%$ & $0 \%$ & $75 \%$ & $75 \%$ & $0 \%$ \\
\hline Ajuste de componentes & $5 \%$ & $5 \%$ & $0 \%$ & $37 \%$ & $0 \%$ & $26 \%$ & $53 \%$ & $11 \%$ \\
\hline Área de trabalho & $9 \%$ & $27 \%$ & $0 \%$ & $0 \%$ & $0 \%$ & $36 \%$ & $45 \%$ & $27 \%$ \\
\hline Armazenamento & $67 \%$ & $0 \%$ & $100 \%$ & $0 \%$ & $0 \%$ & $0 \%$ & $0 \%$ & $0 \%$ \\
\hline Equipamentos/ferramentas & $0 \%$ & $100 \%$ & $0 \%$ & $0 \%$ & $0 \%$ & $0 \%$ & $0 \%$ & $0 \%$ \\
\hline Instalações provisórias & $0 \%$ & $0 \%$ & $50 \%$ & $0 \%$ & $50 \%$ & $0 \%$ & $0 \%$ & $0 \%$ \\
\hline Proteção & $17 \%$ & $33 \%$ & $17 \%$ & $50 \%$ & $0 \%$ & $50 \%$ & $50 \%$ & $0 \%$ \\
\hline Sequenciamento & $3 \%$ & $0 \%$ & $0 \%$ & $59 \%$ & $0 \%$ & $28 \%$ & $10 \%$ & $28 \%$ \\
\hline
\end{tabular}

Fonte: Autoria própria.

A Figura 7 apresenta a porcentagem de casos analisados de acordo com o índice de prioridade a ser considerado para as empresas.

Figura 7 - Porcentagem de casos ocorridos por índice de prioridade

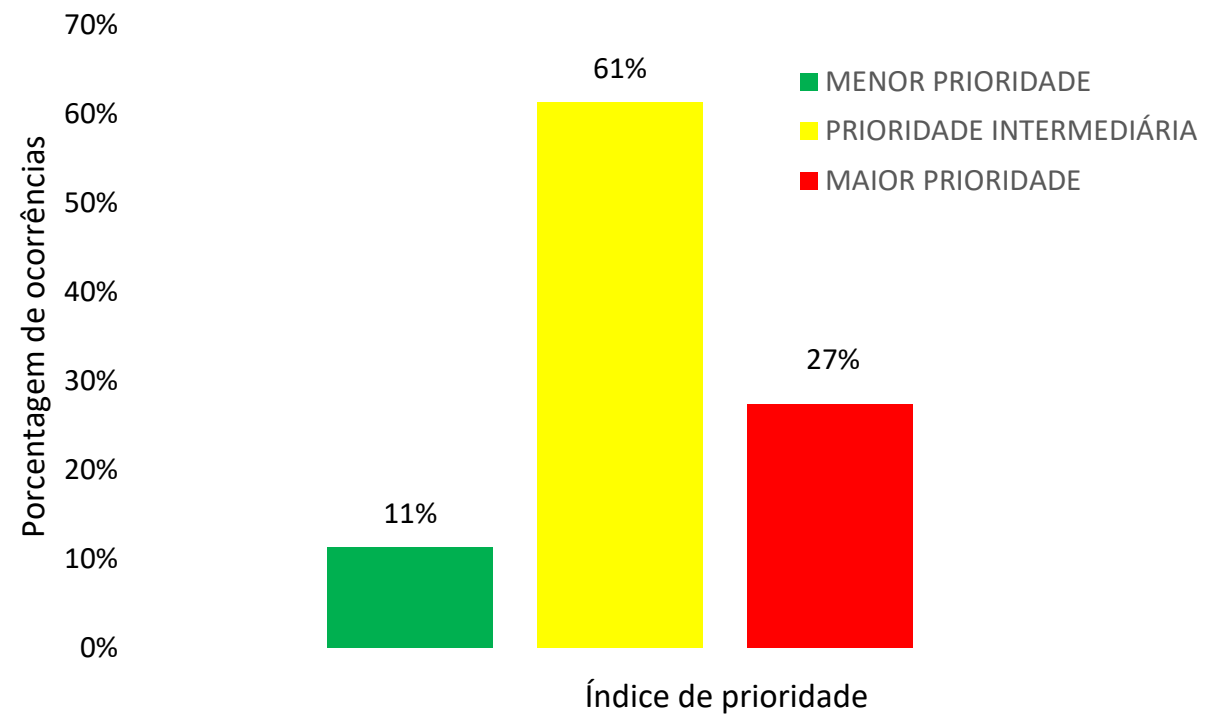

Fonte: Autoria própria.

Destaca-se a ocorrência de inúmeros casos "prováveis de acontecer", mas que não possuem alta severidade. Contudo, esses casos devem ser analisados ao se considerar a repetitividade de ocorrências, que ao final do processo podem gerar problemas maiores e que poderiam ser previstos na etapa de planejamento. 
O estudo realizado na obra $\mathrm{C}$ foi o que resultou em menos casos de improvisação, o que pode ser justificado pelo controle de serviço eficaz realizado pela empresa, e principalmente, e por conseguir atender aos pré-requisitos necessários à execução de cada serviço.

\section{CONCLUSÕES}

A partir dos resultados obtidos no cenário goiano constatou-se novos impactos que devem ser considerados, por influenciarem direta e negativamente no sistema de gestão das empresas: custo e cronograma.

O sequenciamento e o ajuste de componentes são as categorias mais preocupantes do planejamento, pois geram inúmeros impactos à produção. Em relação ao sequenciamento, a causa mais significativa nas três obras pesquisadas relaciona-se à personalização dos apartamentos pelos clientes.

Destaque à segurança, apesar de não ser o impacto mais significativo dos empreendimentos pesquisados, torna-se um ponto de alerta para as empresas, por possuir um risco elevado.

\section{REFERÊNCIAS}

AMARAL, T. G., MARTINS, A. G., DINOAH, L. O., RIBEIRO, R. G. O. S. Determinação das perdas por making-do em empresas goianas. Goiânia, 2018. Trabalho de Conclusão de Curso (Graduação em Engenharia Civil), Universidade Federal de Goiás, Goiânia, 2018.

BOSSINK, B. A. G.; BROUWERS, H. J. H. Construction Waste: quantification and source evaluation, Journal of Construction Engineering Management, v. 122, n. 1, p. 55-60, 1996.

BRAGA, P. B. Análise de perdas por making-do por meio de planilhas dinâmicas. Goiânia, 2018. Trabalho de conclusão de Curso (Graduação em Engenharia Civil), Universidade Federal de Goiás, Goiânia, 2018.

BRANDÃO, C. M., ELIAS, K. V. Identificação de perdas por improvisação em canteiros de obras. Goiânia, 2018. Trabalho de Conclusão de Curso (Graduação em Engenharia Civil), Universidade Federal de Goiás, Goiânia, 2018.

BULHÕES, I. R. Diretrizes Para Implementação de Fluxo Contínuo na Construção Civil: proposta baseada em dois estudos de caso. Campinas, 2009. Tese (Doutorado em Engenharia Ambiental Urbana) Faculdade de Engenharia Civil, Arquitetura e Urbanismo, Universidade Estadual de Campinas, Campinas, 2009.

BURATI, J. L., FARRINGTON, J. J., \& LEDBETTER, W. B. Causes of Quality Deviations in Design and Construction. Journal of Construction Engineering and Management, 118(1), 34-49. 1992.

CHOI, S. et al. Waste Elimination of Mucking Process of a Petroleum Storage Tunnel through the Value Stream Analysis, In: ANNUAL CONFERENCE OF THE INTERNATIONAL GROUP FOR LEAN CONSTRUCTION, 10., Manchester, 2002. Proceedings... Manchester, 2002.

ENSHASSI, A. Materials control and waste on building sites, Building Research \& Information, v. $24 \mathrm{n}$. 1, p. 31-34, 1996.

FIREMAN, M. C. T. Proposta de método de controle integrado entre produção e qualidade com mensuração de perdas por making-do e pacotes informais. Porto Alegre, 2012. 179 f. Dissertação (Mestrado em Engenharia) - Programa de Pós-Graduação em Engenharia Civil, Universidade Federal do Rio Grande do Sul, Porto Alegre, 2012.

FIREMAN, M. C. T. et al. Integrating Production and Quality Control: monitoring making-do and unfinished work. In: ANNUAL CONFERENCE OF THE INTERNATIONAL GROUP FOR LEAN CONSTRUCTION, 21th, Fortaleza, 2013. Proceedings... Fortaleza, 2013.

FORMOSO, C. T. et al. An Exploratory Study on the Measurement and Analysis of Making-Do in Construction Sites. In: ANNUAL CONFERENCE OF THE INTERNATIONAL GROUP FOR LEAN CONSTRUCTION, 19th , Lima, 2011. Proceedings... Lima, 2011. 
FORMOSO, C. T. et al. Material Waste in Building Industry: main causes and prevention. Journal of construction engineering and management, v. 128, n. 4, p. 316-325, 2002.

HAN, S. et al. Identification and Quantification of Non-Value Adding Effort Due to Errors and Changes in Design and Construction Projects, Journal of Construction Engineering and Management, v. 1 n. 1, p. 291, 2011.

HWANG, B. G. et al. Measuring the Impact of Rework on Construction Cost Performance. Journal of Construction Engineering and Management, v. 135, n. 3, p. 187-198, 2009

KOSKELA, L. An Exploration Towards a Production Theory and its Application to Construction. Thesis (Ph.D) - Technical Research Centre of Finland, Espoo, 2000.

KOSKELA, L. Making-do - The Eighth Category of Waste. In: CONFERENCE OF THE INTERNATIONAL GROUP FOR LEAN CONSTRUCTION, 12, 2004, Dinamarca.

Proceedings...Dinamarca, 2004.

KOSKENVESA, A. et al. Waste and Labor Productivity in Production Planning. In: ANNUAL CONFEENCE OF THE INTERNATIONAL GROUP FOR LEAN CONSTRUCTION, 18., Haifa, 2008. Proceedings... Haifa, 2008.

LOVE, P; EDWARDS, D.J. Determinants of Rework in Building Construction Projects. Engineering, Construction and Architectural Management, v. 11, n. 4, p. 259-274, 2004.

LOVE, P. Influence of Project Type and Procurement Method on Rework Costs in Building Construction Projects. Journal of Construction Engineering Management, v. 128, n. 1, p. 18-29, 2002.

MONDEN, Y. Toyota Production System: Practical Approach to Production Management. Norcross, USA, Industrial Engineering and Management Press, 1983.

NAHMENS, I.; IKUMA, L. H. Effects of Lean on Sustainability of Modular Homebuilding. Journal of Architectural Engineering, v. 1, n. 1, p. 25, 2011

NAHMENS, I.; MULLENS, M. A. Lean Homebuilding: lessons learned from a precast concrete panelizer. Journal of Architectural Engineering, v. 17, n. 4, p. 155, 2011.

OHNO, T. Toyota Production System. Portland: Productivity Press, 1988.

SANTOS, P. R. R.; SANTOS, D. de G. Investigação de perdas devido ao trabalho inacabado e o seu impacto no tempode ciclo dos processos construtivos. Ambiente Construído, Porto Alegre, v. 17, n. 2, p. 39-52, abr./jun. 2017.

SENARATnE, S.; SEXTON, M. G. Role of Knowledge in Managing Construction Project Change. Engineering, Construction and Architectural Management, v. 16, n. 2, p. 186- 200, 2009.

SKOYLES, E.R. Site accouting for waste of materials. Building Research Establishment, July/Aug. 1976

SOMMER, L. Contribuições para um método de identificação de perdas por improvisação em canteiros de obras. Porto Alegre, 2010. 150 f. Dissertação (Mestrado em Engenharia) - Programa de PósGraduação em Engenharia Civil, Universidade Federal do Rio Grande do Sul, Porto Alegre, 2010.

VIANA, D. D.; FORMOSO, C. T.; KALSAAS, B. T. Waste in Construction: a systematic literature review on empirical studies. In: CONFERENCE OF THE INTERNATIONAL GROUP FOR LEAN CONSTRUCTION, 20., San Diego, 2012. Proceedings... San Diego, 2012.

WOMACK, J.P.; JONES, DT. A mentalidade enxuta nas empresas - Elimine o desperdício e crie riqueza. Rio de janeiro: Elsevier, 2004.

YU, H. et al. Development of Lean Model For House Construction Using Value Stream Mapping. Journal of Construction Engineering and Management, v. 135, n. 8, p. 782-790, 2009.

ZHAO, Z. Y. et al. Prediction System For Change Management in Construction Project. Journal of Construction Engineering Management, v. 136, n. 6, p. 659-669, 2010. 
SIBRAGEC - ELAGEC 2019 - de 23 a 25 de Outubro - LONDRINA - PR

APÊNDICE A

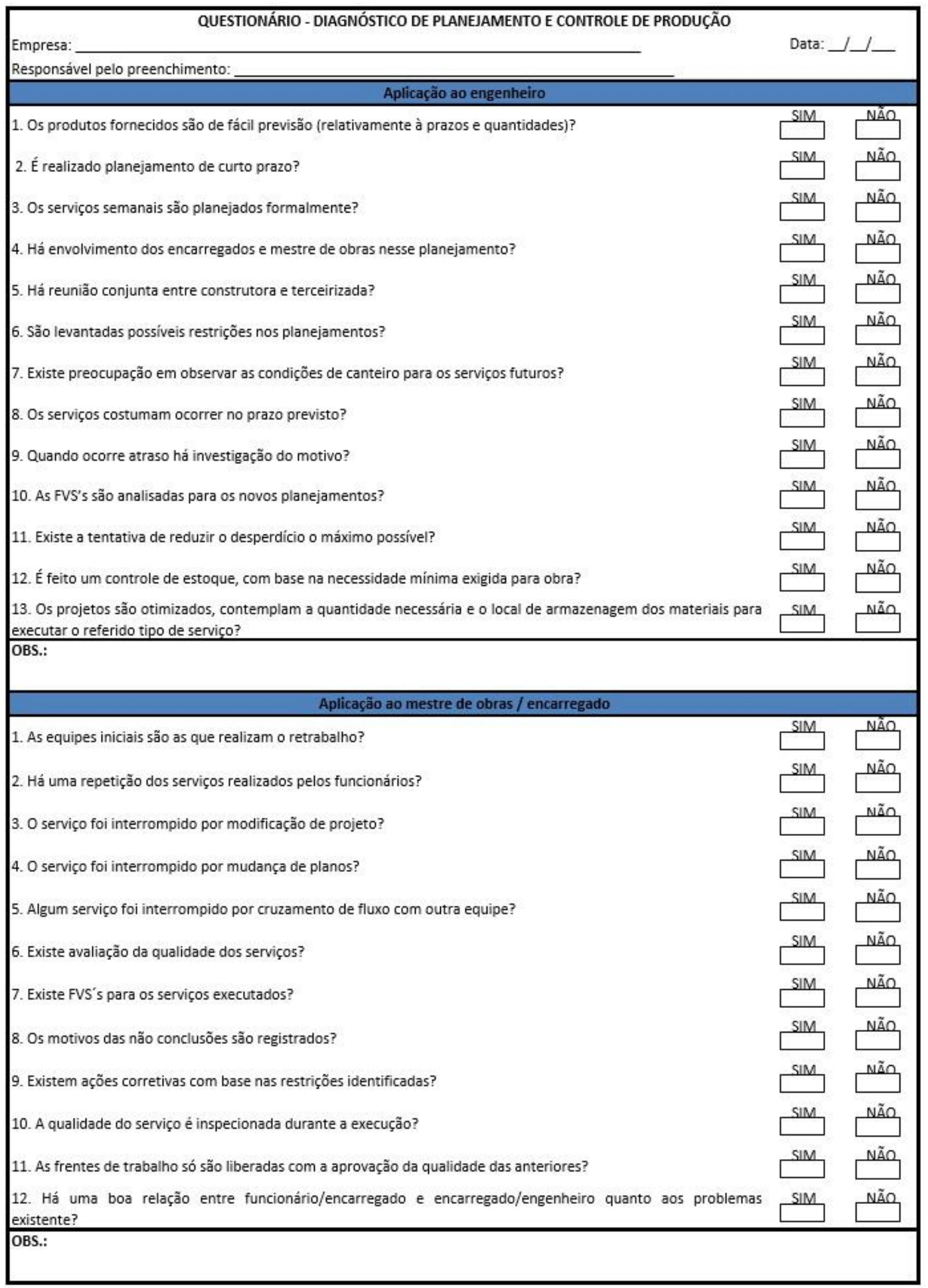

